\title{
CT imaging findings of abdominopelvic vascular compression syndromes: what the radiologist needs to know
}

\author{
Cecilia Gozzo ${ }^{1,2}$, Dario Giambelluca ${ }^{1}$, Roberto Cannella ${ }^{1,2^{*}}$ D , Giovanni Caruana ${ }^{1}$, Agita Jukna ${ }^{3,4}$, Dario Picone ${ }^{1}$, \\ Massimo Midiri ${ }^{1}$ and Giuseppe Salvaggio ${ }^{1}$
}

\begin{abstract}
Abdominopelvic vascular compression syndromes include a variety of uncommon conditions characterized by either extrinsic compression of blood vessels by adjacent anatomical structures (i.e., median arcuate ligament syndrome, nutcracker syndrome, May-Thurner syndrome) or compression of hollow viscera by adjacent vessels (i.e., superior mesenteric artery syndrome, ureteropelvic junction obstruction, ureteral vascular compression syndromes, portal biliopathy). These syndromes can be unexpectedly diagnosed even in asymptomatic patients and the predisposing anatomic conditions can be incidentally discovered on imaging examinations performed for other indications, or they can manifest with atypical abdominal symptoms and acute complications, which may lead to significant morbidity if unrecognized. Although computed tomography (CT) is an accurate noninvasive technique for their detection, the diagnosis remains challenging due to the uncommon clinical presentation and often overlooked imaging features. Dynamic imaging may be performed in order to evaluate patients with inconstant symptoms manifesting in a specific position. The purposes of this paper are to review the $C T$ imaging findings of abdominopelvic vascular compression syndromes, correlating with anatomical variants and to provide key features for the noninvasive imaging diagnosis.
\end{abstract}

Keywords: Computed tomography, Abdomen, Vascular compression syndrome, Vascular syndromes

\section{Key points:}

- Abdominopelvic vascular compression syndromes present with a wide spectrum of clinical manifestations.

- Contrast-enhanced CT allows the identification of typical imaging features and possible complications.

\footnotetext{
*Correspondence: rob.cannella89@gmail.com

'Sezione di Scienze Radiologiche, Biomedicina, Neuroscienze e Diagnostica avanzata (BIND), University of Palermo, Via del Vespro 129, 90127 Palermo, Italy

2Department of Medical Surgical Sciences and Advanced Technologies "G.F. Ingrassia"-Radiology I Unit, University Hospital "Policlinico Vittorio Emanuele", Catania, Italy

Full list of author information is available at the end of the article
}

- Predisposing anatomical conditions are encountered on CT examinations performed for unrelated indications.

\section{Introduction}

Abdominopelvic vascular compression syndromes include a large spectrum of abdominal conditions (Table 1) characterized by either extrinsic compression of blood vessels by adjacent anatomical structures (i.e., median arcuate ligament syndrome, nutcracker syndrome, MayThurner syndrome) or compression of hollow viscera by adjacent vessels (i.e., superior mesenteric artery syndrome, ureteropelvic junction obstruction, ureteral vascular compression syndromes, portal biliopathy) [1]. The exact prevalence of these syndromes is not known, but it can be estimated based on the prevalence of 


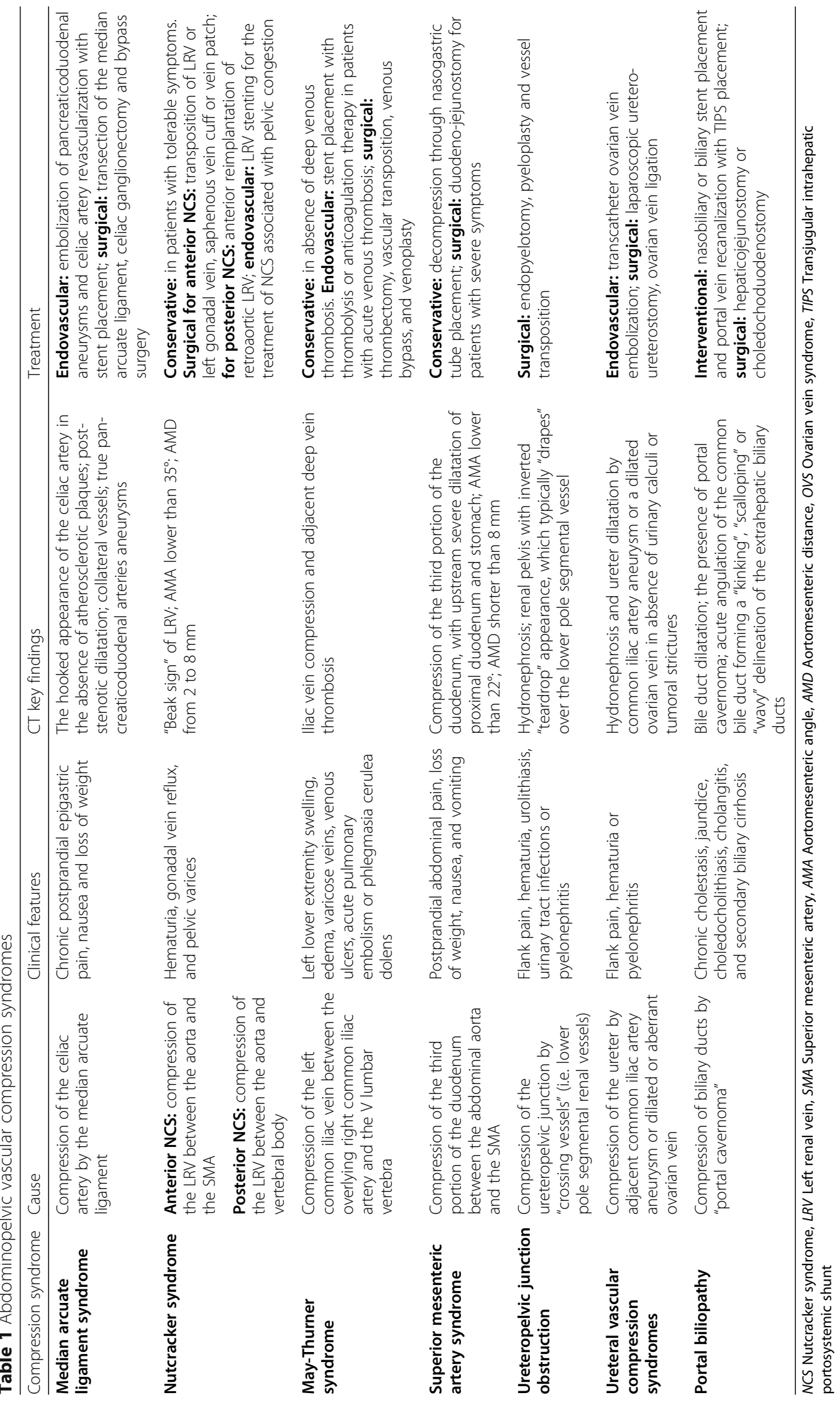


predisposing anatomical variants. Clinically, these syndromes are typically encountered in young and otherwise healthy patients and may manifest with a variety of nonspecific abdominal symptoms, ranging from vague abdominal pain, nausea and vomiting, loss of weight to acute abdominal hemorrhage, arterial ischemia and embolism or venous stasis and thrombosis, making the clinical diagnosis particularly challenging [2]. The predisposing anatomic abnormalities may be incidentally discovered even in asymptomatic patients on abdominal imaging examinations performed for unrelated indications [1]. Their recognition in asymptomatic patients is relevant for preventing vascular injuries during surgical procedures or for prompt treatment of possible complications related to these syndromes (i.e., aneurysm rupture in median arcuate ligament syndrome, deep venous thrombosis and pulmonary embolism in May-Thurner syndrome or urinary tract infections in ureteropelvic junction obstruction).

The noninvasive diagnosis of abdominopelvic vascular compression syndromes may be unexpectedly made with different imaging modalities including ultrasound, computed tomography $(\mathrm{CT})$, and magnetic resonance imaging (MRI). Among them, contrast-enhanced CT is the most frequently recommended imaging method in clinically suspected syndromes, providing accurate detection of vascular structures and their relationship with adjacent organs [1,2]. MRI may be preferred in children or young patients due to the absence of ionizing radiation. Dynamic imaging with Doppler ultrasound or CT allows the evaluation of changes in flow velocity or vessel caliber and may be performed in patients with inconstant symptoms $[2,3]$. The combination of noninvasive imaging modalities, which allow the precise evaluation of anatomical structures, and invasive techniques, such as venography or arteriography that are useful for the direct measurement of pressure gradients, is helpful to establish the definitive diagnosis. However, the invasive procedures should be limited to the most doubtful cases or when the endovascular treatment needs to be performed.

The purpose of this article is to review the pathophysiological and clinical background of abdominopelvic vascular compression syndromes and the most relevant $\mathrm{CT}$ imaging findings for the diagnosis.

\section{Median arcuate ligament syndrome}

Median arcuate ligament syndrome (MALS), also called "Dunbar syndrome", represents a condition characterized by external compression of celiac artery root by the median arcuate ligament (Fig. 1). This latter is a fibrous arch connecting the right and left diaphragmatic crura on either side of aortic hiatus. Two anatomic conditions

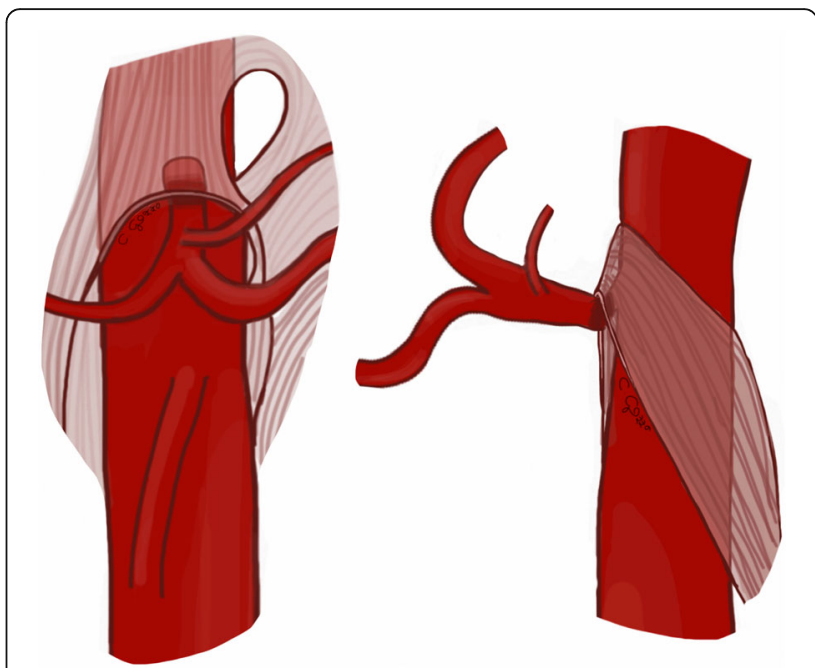

Fig. 1 Drawing illustrating the coronal and sagittal views of the median arcuate ligament compressing the root of the celiac artery

can predispose to MALS: the higher origin of the celiac artery or the lower insertion of median arcuate ligament [3]. These anatomical variants are present in about 10$24 \%$ of the general population [3,4]. Overall, MALS is more commonly encountered in young thin women $[2$, $3]$.

The classic clinical manifestations of MALS include chronic postprandial epigastric pain, nausea, and loss of weight due to dynamic compression of the celiac artery [1]. However, this anatomical anomaly is asymptomatic in up to $85 \%$ of patients and may be incidentally encountered on CT examinations performed for unrelated reasons $[1,5,6]$. The mechanism of pain is still debated. During expiration, the abdominal aorta and its branches are displaced superiorly and the median arcuate ligament compression of the celiac artery increases. This may be responsible for a steal phenomenon with blood flow diverted away from the superior mesenteric artery to the celiac artery branches trough the collateral pathway of pancreaticoduodenal arcades [1, 4]. Epigastric pain may be also related to celiac plexus nerve compression [7]. The development of collateral circulation through the pancreaticoduodenal arcades prevents chronic hypoperfusion in patients with severe stenosis of the celiac artery [6].

In children and young adults, the Doppler ultrasound examination may directly demonstrate the dynamic variations of flow velocity in the celiac artery during respiratory excursions. Indeed, celiac artery compression at the end of expiration markedly increases the flow velocity [8]. Dynamic CT examination may also be performed in both deep inspiration and expiration in order to evaluate the dynamic modifications in celiac artery diameter [1]. CT imaging should include the early arterial phase 
acquired in deep expiration in order to increase the proximal celiac trunk compression by the median arcuate ligament, followed by the portal venous phase in deep inspiration [9]. Sagittal and coronal images should be included for optimal visualization of the celiac artery. The proximal narrowing of the celiac trunk can be better depicted on sagittal CT reconstructions, demonstrating a focal indentation on the superior surface of the vessel with a typical "hooked appearance" (Fig. 2), in the absence of atherosclerotic plaques or other causes of extrinsic compression. However, calcified plaques may coexist in older patients and make the differential diagnosis of celiac artery stenosis challenging. In these cases, the lack of hooked appearance on sagittal CT images is the only feature to rule out the presence of MALS [5]. Post-stenotic dilatation and development of peripancreatic collaterals can also be visualized on CT images, and they are imaging features suggesting the proximal compression of the celiac artery. Sugae et al. [10] proposed a grading system of celiac artery stenosis caused by median arcuate ligament compression based on stenosis grade, length of stenosis, distance from the abdominal aorta and presence of small collateral vessels found on CT angiography. Severe stenosis of the celiac artery may be particularly relevant in patients undergoing pancreatic surgery with pancreaticoduodenectomy and it is a significant risk factor for upper abdominal organs infarction [11]. In these patients, the celiac artery remains the only vessel for the arterial blood supply of the upper abdominal organs due to the transection of the pancreaticoduodenal artery during the surgical procedure.

The most concerning consequence of MALS is the visceral artery aneurysm formation. Severe celiac artery stenosis may lead to altered hemodynamic in the pancreaticoduodenal arcades, causing intimal damage and dysfunction of the medial arterial layer with possible formation of true pancreaticoduodenal arteries aneurysms (Fig. 3). Aneurysm formation should be carefully ruled out as it is correlated with a high possibility of rupture and hemorrhage, developing in up to $40 \%$ of patients, with a mortality rate of $50 \%[3,4,12]$.

The symptomatic MALS may be managed with surgical transection of the median arcuate ligament, celiac ganglionectomy, and bypass surgery, which may provide sustained symptom relief [7]. Endovascular treatments include embolization of pancreaticoduodenal aneurysms and celiac artery revascularization with stent placement prior to surgical decompression [4]. Stent placement alone is contraindicated due to the risk of occlusion caused by sustained extrinsic compression from the median arcuate ligament [4].

\section{Nutcracker syndrome}

Nutcracker syndrome (NCS) is defined by the compression of the left renal vein (LRV) between the aorta and the superior mesenteric artery (SMA), known as "anterior nutcracker syndrome" (Fig. 4), or more rarely between the aorta and a vertebral body, recognized as "posterior nutcracker" or "pseudo-nutcracker syndrome" (Fig. 5) [13, 14]. The definite prevalence of NCS is unclear due to the high variability in clinical manifestations $[15,16]$. Some anatomical variants may cause NCS. The retroaortic LRV, an anatomical variant seen in about 3\% of the population, can be responsible for pseudonutcracker syndrome in $9 \%$ of patients with left-sided varicocele [17]. The "renal collar", also known as
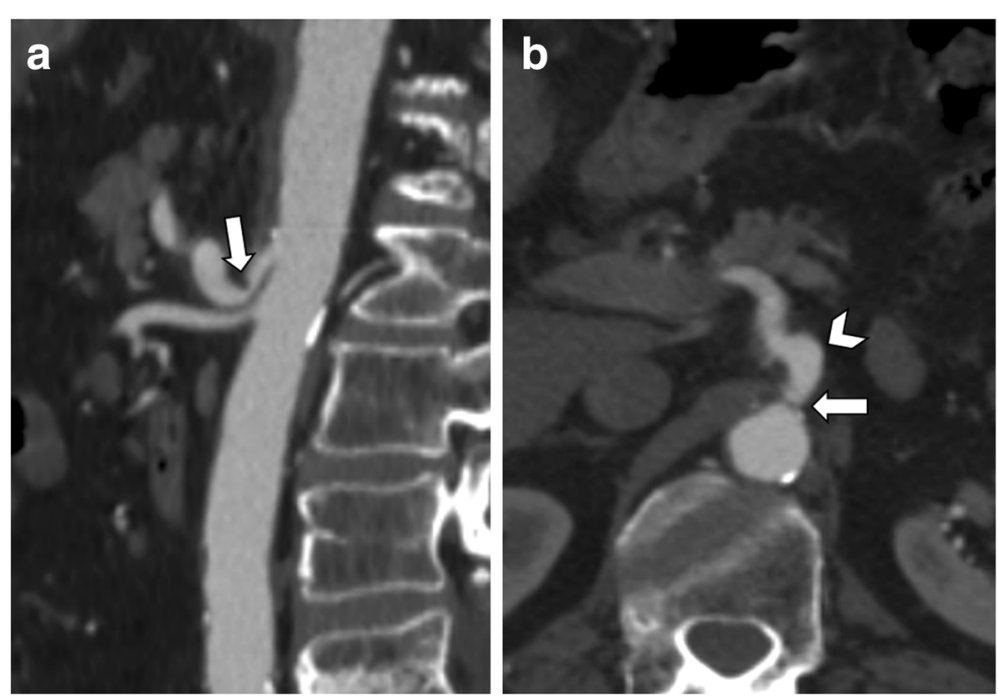

Fig. 2 A 65-year-old man with median arcuate ligament compressing the celiac artery. a Sagittal CT image shows the "hooked appearance" (arrow) with severe stenosis at the origin of the celiac artery, in the absence of atherosclerotic plaques. b Corresponding axial CT image shows celiac artery compression (arrow) with post-stenotic dilatation (arrowhead) 

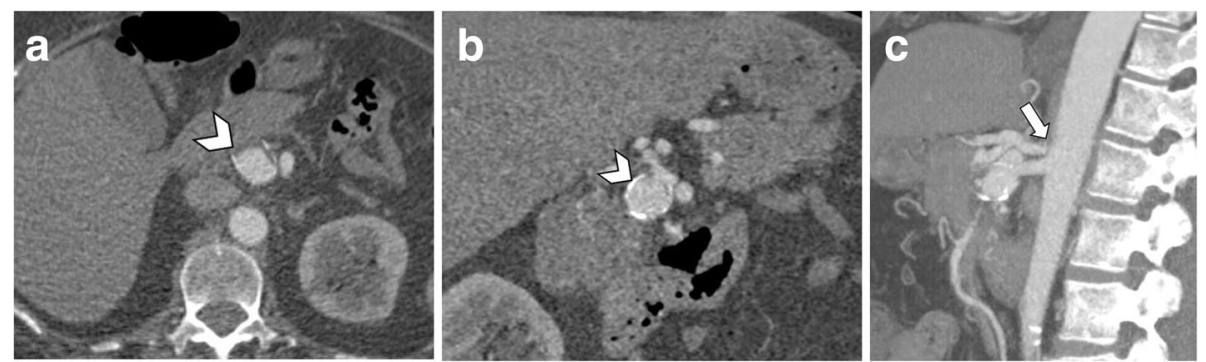

Fig. 3 A 63-year-old woman with median arcuate ligament compressing the celiac artery. Axial (a) and coronal (b) arterial phase CT image shows pancreaticoduodenal artery aneurysm (arrowheads). Reconstructed MIP images on the sagittal plane (c) show severe stenosis at the origin of the celiac artery (arrow) with post-stenotic dilatation on arterial phase images acquired during expiration

"circumaortic venous ring", is another anatomic variant characterized by the presence of two LRVs passing anteriorly and posteriorly to the aorta, respectively [17]. In patients with "renal collar" ( $0.3 \%$ to $16 \%$ of the population), the concomitant compression of the anterior LRV-between the abdominal aorta and SMA - and the posterior LRV-between the abdominal aorta and spine-is defined as "combined nutcracker syndrome" [15]. Anterior NCS may coexist with SMA syndrome due to their common pathogenesis [1]. Rapid loss of weight and retroperitoneal fat, ptosis of the left kidney, preaortic fibrous tissue, and duodenal interposition have been reported as predisposing factors for NCS $[1,13]$.

Nutcracker syndrome may lead to increased venous pressure in the left kidney, resulting in rupture of glomerular capillaries with intermittent hematuria, gonadal vein reflux, and formation of pelvic varices [1]. However, when this anatomic compression is only seen on imaging

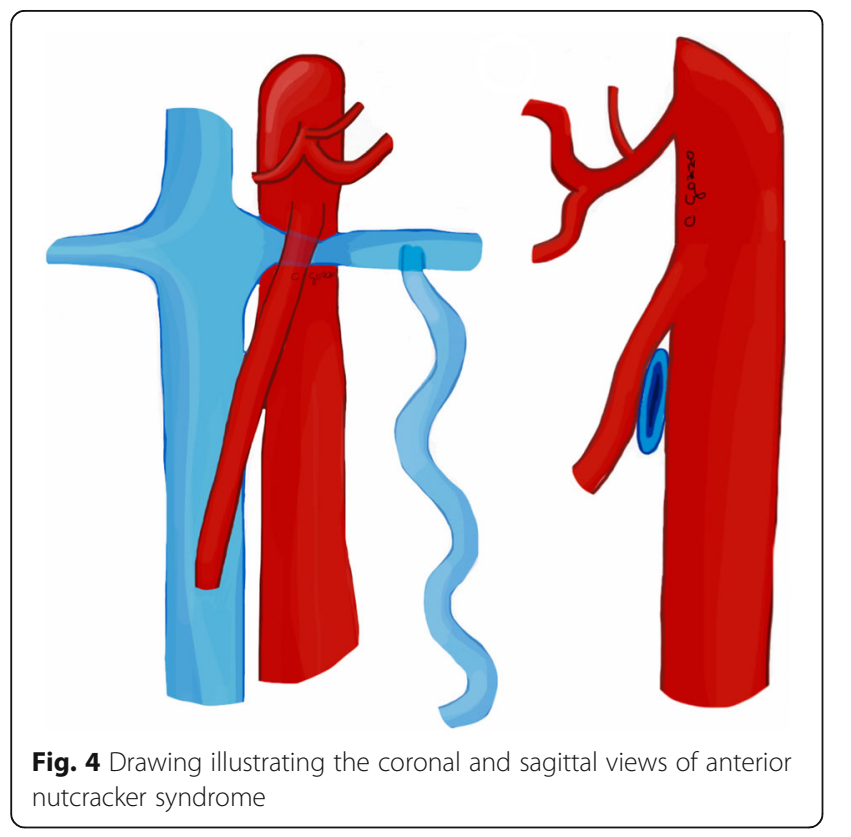

without clinical symptoms, it should be labeled as "nutcracker phenomenon" [1].

The preaortic or retroaortic course of the LRV and the compression between the abdominal aorta and the SMA can be accurately visualized on $\mathrm{CT}$ images acquired on arterial or portal venous phases (Fig. 6) [18]. Axial CT images show the typical "beak sign" of LRV (Fig. 7a), which consists of an abrupt narrowing of the LRV with an acute angle below the aortomesenteric junction [15, 18]. Sagittal CT images allow to calculate the aortomesenteric angle (AMA) between SMA and abdominal aorta, which normally ranges from $38^{\circ}$ to $56^{\circ}$ [19]. If the AMA decreases to $9-35^{\circ}$, external compression of both the duodenum (SMA syndrome, see below) and left renal vein (NCS) may occur $[19,20]$. Indeed, the diagnosis of anterior NCS may be made when AMA is less than $35^{\circ}$ [21]. Moreover, the aortomesenteric distance shows a decrease from a normal value of $10-28 \mathrm{~mm}$ to $2-8$ $\mathrm{mm}[1,20]$. CT can also depict the resulting gonadal vein dilatation and pelvic varices (Fig. 7), although it does not allow to measure flow velocity and direction [15]. Color Doppler ultrasound may demonstrate a

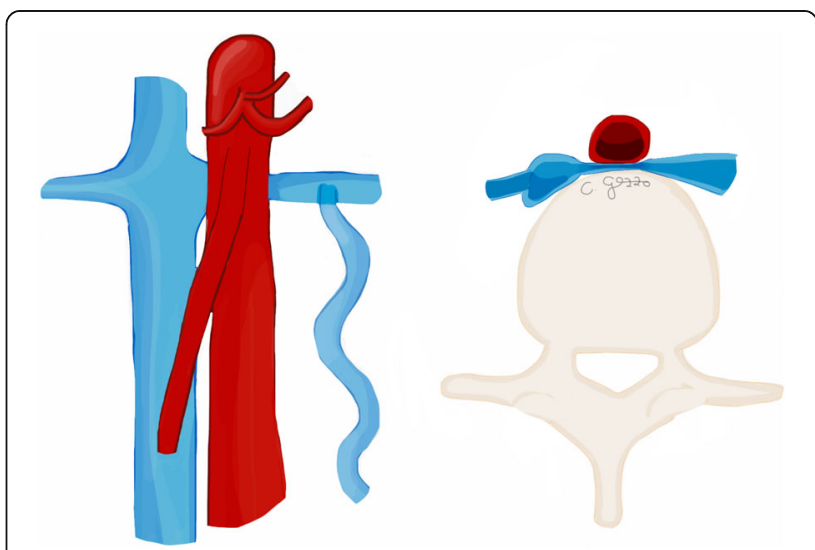

Fig. 5 Drawing illustrating the coronal and axial views of posterior nutcracker syndrome 


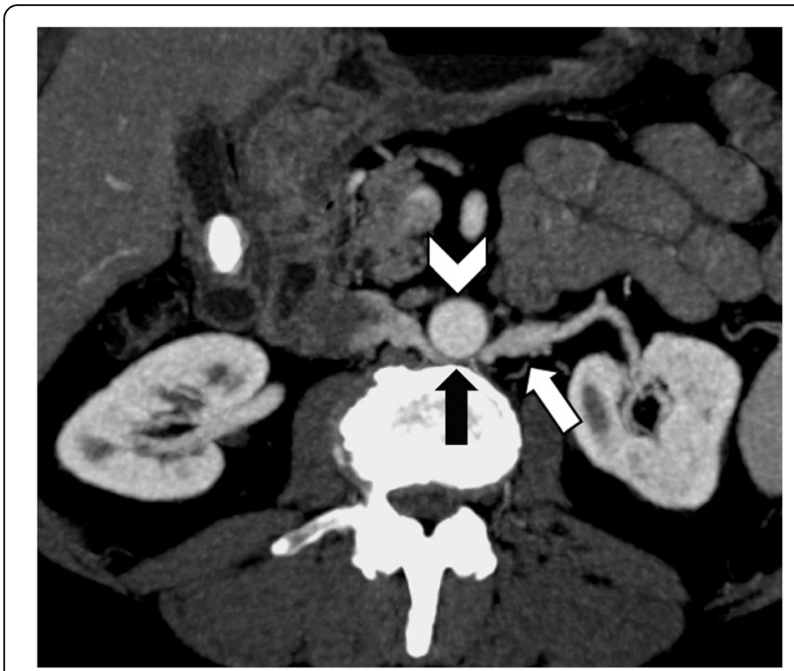

Fig. 6 A 78-year-old man with posterior nutcracker syndrome. Axial CT image on the arterial phase shows left renal vein (white arrow) compressed between the aorta (arrowhead) and the vertebral body (black arrow)

significantly increased peak systolic velocity ratio between the point of compression and the hilar LRV [1, 15]. Despite being more invasive, retrograde venography depicts the level of compression and allows the determination of the reno-caval pressure gradient, which is diagnostic for NCS over $3 \mathrm{mmHg}[15,22]$.

Management of NCS can be either conservative and observational in patients with mild hematuria and tolerable symptoms or invasive in case of recurrent gross hematuria, severe symptoms or ineffective conservative treatment $[15,23]$. Invasive treatments include open surgical and endovascular interventions [24]. Surgical treatment of anterior NCS consists in the transposition of LRV or left gonadal vein in the inferior vena cava, saphenous vein cuff, or vein patch, while anterior reimplantation of retroaortic LRV into the inferior vena cava is performed in posterior NCS [24]. Finally, endovascular LRV stenting may be considered for the treatment of NCS associated with pelvic congestion $[15,25]$.

\section{May-Thurner syndrome}

May-Thurner syndrome (MTS), also described with the eponymous of "Cockett syndrome", refers to the chronic compression of the left common iliac vein between the overlying right common iliac artery, anteriorly, and the fifth lumbar vertebra, posteriorly (Fig. 8) [2]. Variants of MTS include the right common iliac vein compression by the right common iliac artery or the left iliac vein compressed by the left common iliac artery [26]. MTS is mainly discovered in women between the 2nd and 4th decades of life $[1,14]$. The exact prevalence of MTS is unknown, but it is assessed to occur in $2-5 \%$ of patients presenting with lower extremity venous disorders [27].

MTS can lead to chronic venous stasis, determining left iliac and femoral veins thrombosis, clinically manifesting with unilateral left lower extremity swelling, edema, varicose veins, venous ulcers, and other potential severe complications, like acute pulmonary embolism or phlegmasia cerulea dolens $[1,2]$. This latter is characterized by blue and painful leg, due to massive deep venous thrombosis combined with arterial occlusion determined by concomitant compartment syndrome [28]. Although venous obstruction is commonly caused by extrinsic factors (physical compression of the left common iliac vein), it could also be secondary to intrinsic factors, particularly to endothelial damage due to chronic pulsatile force of the overlying right common iliac artery $[1,29]$.

Doppler ultrasound may depict the presence of deep venous thrombosis of the left lower limb. As opposed, left iliac vein thrombosis may be challenging to diagnose because this vessel lies deep in the pelvis and may be concealed by gas-filled intestinal loops [1]. Additional imaging with contrast-enhanced CT should be required in the case of suspected MTS [29]. Contrast-enhanced CT images acquired after the intravenous administration of contrast agent on venous and delayed phases are often sufficient to visualize the iliac vein compression and adjacent deep vein thrombosis (Fig. 9). Moreover, CT helps to exclude other conditions such as pelvic masses or lymphadenopathy compressing the left iliac vein or

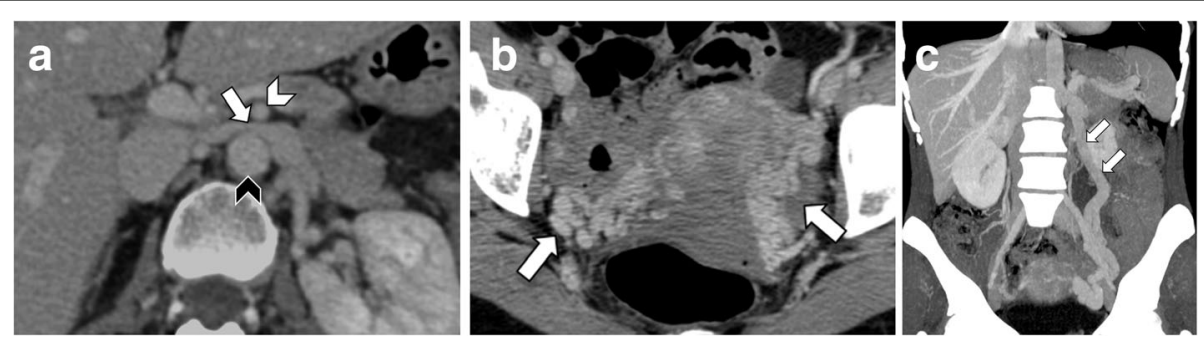

Fig. 7 A 50-year-old woman with anterior nutcracker syndrome. a Axial contrast-enhanced CT image on the portal venous phase shows the typical "beak sign" (arrow) with left renal vein compression between the aorta (black arrowhead) and superior mesenteric artery (white arrowhead). $\mathbf{b}$ CT image at the level of the pelvis demonstrates multiple pelvic varices (arrows). c Coronal reformatted CT MIP image shows marked dilatation of the left ovarian vein (arrows) 


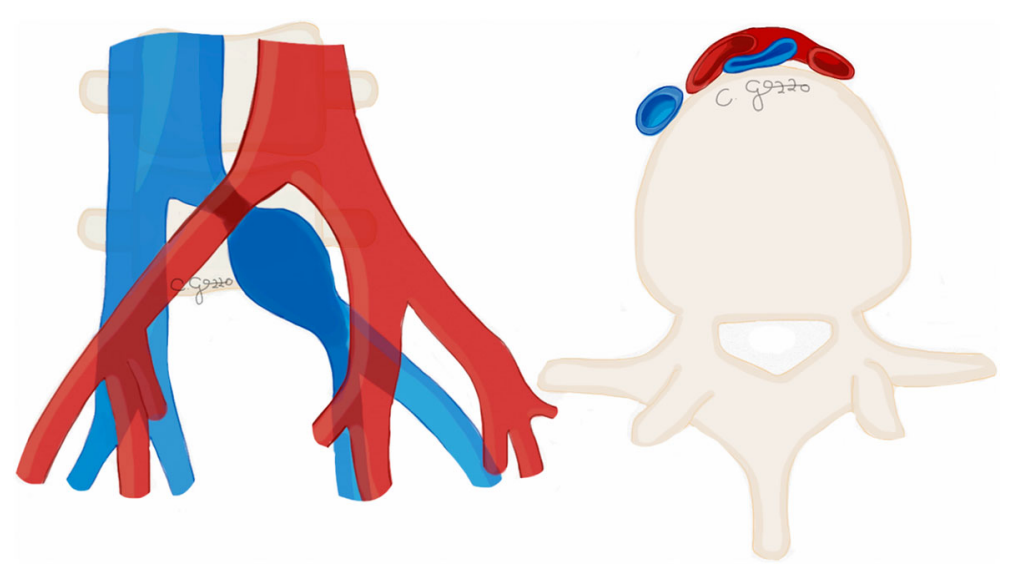

Fig. 8 Drawing illustrating the coronal and axial views of May-Thurner syndrome

inferior vena cava $[1,5]$. Ascending femoral or iliac venography may be performed to confirm the diagnosis in most difficult cases and to measure the pressure gradients, although this is an invasive procedure [14].

Conservative treatments with compression stockings can be sufficient in the absence of deep venous thrombosis. In the case of symptoms, endovascular stent placement is considered the treatment of choice to relieve the mechanical obstruction [1]. Thrombolysis or anticoagulation therapy with compression stockings are only indicated in patients with acute venous thrombosis $[1,14,30]$. An open surgical thrombectomy is exceptional and the only indication is the failure of endovascular therapies [27]. Other surgical options include vascular transposition, venous bypass procedure, and venoplasty with excision of intraluminal bands [30].

\section{Superior mesenteric artery syndrome}

Superior mesenteric artery syndrome, otherwise known as "Wilkie syndrome", is caused by a compression of the third portion of the duodenum between the abdominal aorta and the superior mesenteric artery itself, resulting in duodenal obstruction (Fig. 10) [1]. The prevalence of SMA syndrome is around $0.0024-0.03 \%$ and it may coexist with anterior NCS due to the common pathogenesis, as detailed above [31]. Congenital or acquired anatomic abnormalities with reduction of aortomesenteric angle or loss of normal perivascular fatty cushion are the most common etiologies of SMA syndrome. A congenital abnormal low origin of the SMA or a high attachment of the angle of Treitz, that displaces the duodenum cranially, may be predisposing conditions [32]. Similarly to NCS, severe weight loss and low body mass index are acquired predisposing factors, due to the loss of the normal mesenteric adipose tissue around the superior mesenteric artery, which normally acts as a fatty cushion [5]. Other acquired abnormalities may be associated with surgery, spinal trauma, burns, eating disorders, neoplastic diseases, and malabsorption states [19, 32].

The clinical signs and symptoms of SMA syndrome include duodenal obstruction manifesting with postprandial abdominal pain, loss of weight, nausea, and vomiting due to the slower gastric emptying. Usually, the SMA

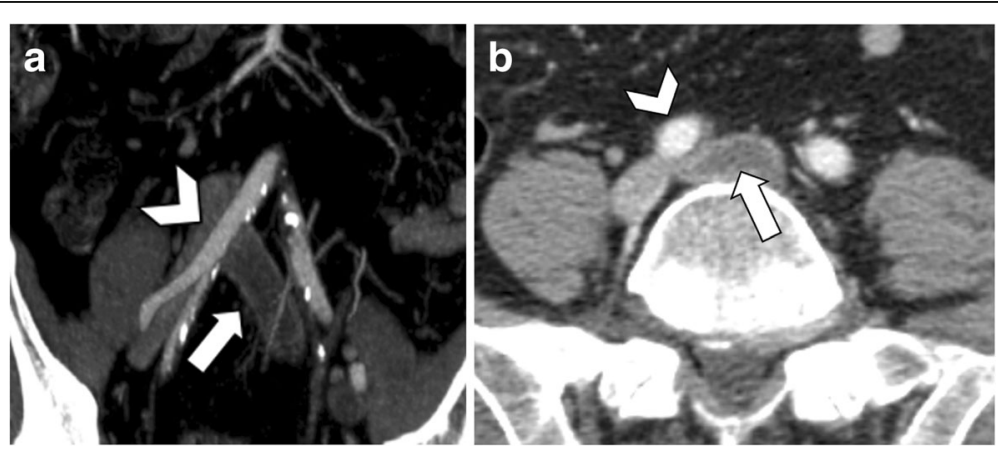

Fig. 9 An 85-year-old man with May-Thurner syndrome. Coronal (a) and axial (b) contrast-enhanced CT images show thrombosed left common iliac vein (arrows) compressed between the right common iliac artery (arrowheads) and fifth lumbar vertebra 


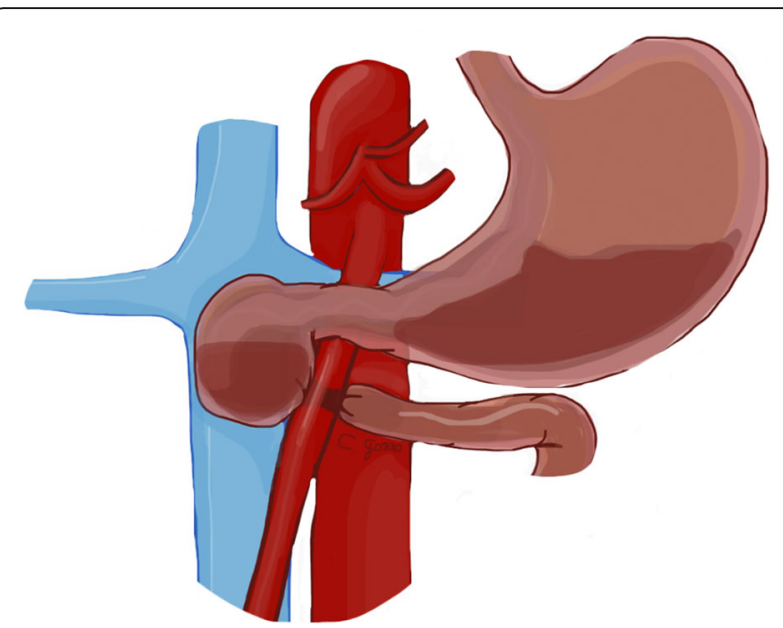

Fig. 10 Drawing illustrating the coronal view of superior mesenteric artery syndrome

syndrome is a diagnosis of exclusion, made when high clinical suspicion is followed by imaging confirmation.

The typical findings of SMA syndrome on CT imaging are the compression of the third portion of the duodenum, with upstream severe dilatation of the proximal duodenum and the stomach (Fig. 11a) [1]. As detailed above, sagittal CT images allow to measure accurately the aortomesenteric angle and distance on the arterial phase $[19,20]$. CT cut-offs for the diagnosis of SMA syndrome are an AMA lower than $22^{\circ}$ and an aortomesenteric distance shorter than $8 \mathrm{~mm}$ [5] (Fig. 11b). Differential diagnosis that can be ruled out with contrastenhanced CT includes other causes of megaduodenum, such as small bowel obstruction, annular pancreas, tumors, inflammatory lesions, aneurysms, or mesenteric ischemia [33, 34].

The management of SMA syndrome is usually conservative and includes decompression through nasogastric tube placement to restore a normal aortomesenteric distance and relieve the obstruction [1]. In the case of failure of conservative management, surgical duodenojejunostomy is indicated for patients with severe symptoms [5].

\section{Ureteropelvic junction obstruction}

Ureteropelvic junction obstruction (UPJO) refers to functional or anatomic obstruction of the upper urinary tract at the confluence of the renal pelvis with the upper part of the ureter. Several causes, congenital or acquired, are implicated in UPJO, most commonly "crossing vessels" passing above the ureteric transition point. Particularly, the lower pole segmental renal vessels (artery or vein) may arise from the main renal artery or vein, or branch as accessory vessels directly from the abdominal aorta, iliac artery, or inferior vena cava [1]. Lower-pole segmental artery can bow anteriorly (Fig. 12) or posteriorly over the ureter. Lower pole crossing vessels are found in about $20 \%$ of healthy patients, while their incidence is up to $45 \%$ in patients with UPJO [35].

UPJO usually remains asymptomatic and it may be incidentally discovered. However, if left untreated, it may manifest with flank pain due to hydronephrosis and can be complicated by hematuria, urolithiasis, urinary tract infections, or pyelonephritis [36]. Secondary UPJO from iatrogenic injuries, inflammation, or neoplastic masses should be considered in the differential diagnosis [36].

Contrast-enhanced $\mathrm{CT}$ allows accurate visualization of the relationship between the crossing vessels and the ureteropelvic junction, as well as the severity of the hydronephrosis. Contrast-enhanced CT should include arterial, portal venous, and excretory phases to identify both arteries and veins as well as their relationship with the renal pelvis or ureter [1]. CT imaging features include dilatation of the renal pelvis with a peculiar inverted "teardrop" appearance, which typically "drapes"
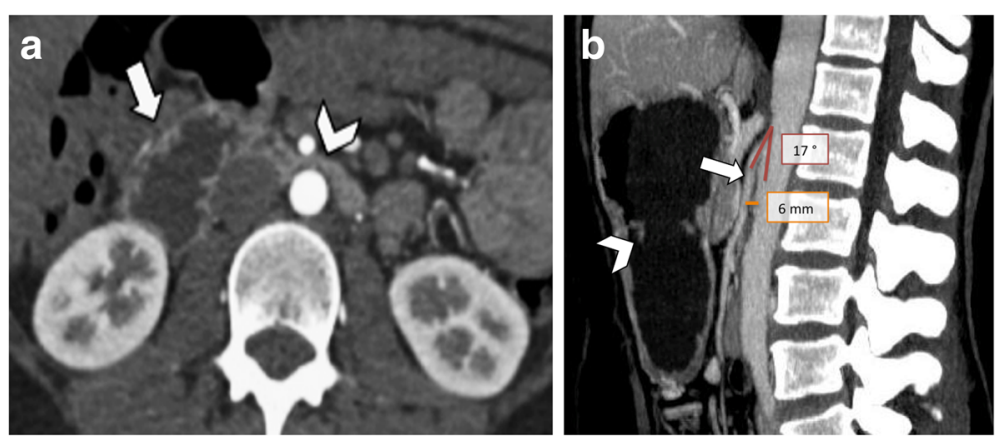

Fig. 11 A 48-year-old woman with superior mesenteric artery syndrome. a Axial CT image on the arterial phase shows dilatation of the second portion of the duodenum (arrow), due to the compression of the third portion (arrowhead) between the abdominal aorta and the SMA. $\mathbf{b}$ Sagittal CT image of the abdomen shows gastric dilatation (arrowhead) caused by compression of the third portion of the duodenum between the abdominal aorta and the SMA (arrow). This sagittal reconstruction also allows measurement of aortomesenteric angle $\left(17^{\circ}\right)$ and aortomesenteric distance $(6 \mathrm{~mm})$, values diagnostic for mesenteric artery syndrome 


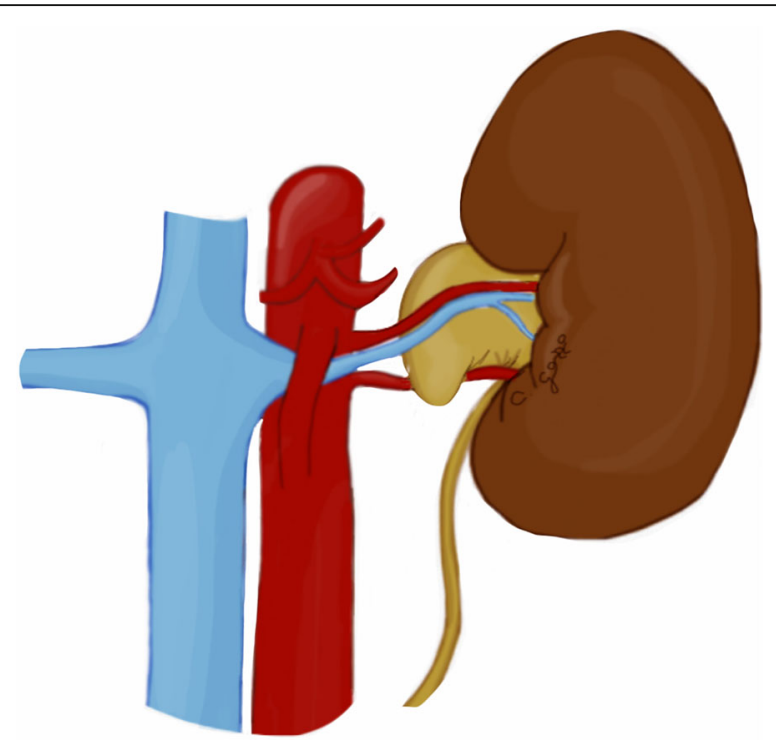

Fig. 12 Drawing illustrating the coronal view of ureteropelvic junction obstruction

over the lower pole segmental artery or vein, while the proximal ureter hooks over the blood vessel (Fig. 13) [1]. Images reconstructed through an oblique sagittal plane, known as "hilar clock-face view", obtained orthogonal to the renal hilum, can better highlight the radial spread of the renal vessels and their relationship to the ureter (Fig. 13b) [36]. The diagnosis of UPJO is particularly relevant for surgical planning since renal arteries are terminal vessels which must be preserved during surgery. Preoperative CT detection of crossing vessels may avoid their accidental iatrogenic injury [1].

Treatment depends on clinical symptoms, the grade of hydronephrosis, and decline in renal function. Surgical treatment of UPJO includes endopyelotomy, pyeloplasty, and vessel transposition [1,37]. Endopyelotomy could be carried out in the absence of crossing vessels, whereas laparoscopic dismembered pyeloplasty is the treatment of choice if crossing vessels are present [1]. In some patients, vessel transposition without dismembered pyeloplasty may be curative [37].

\section{Ureteral vascular compression syndromes}

Obstructive uropathy by vascular compression may also appear at the point of attachment of the ureter with retroperitoneal vessels, such as the ovarian vein, more infrequently testicular vein or common iliac artery. The ureter may even result in compressed by adjacent common iliac artery aneurysm (Fig. 14), especially inflammatory [38] or mycotic aneurysms [39]. The hydroureteronephrosis secondary to a compression by a dilated or aberrant ovarian vein is known as "ovarian vein syndrome" (OVS) (Fig. 15) [1]. OVS is most commonly located on the right side and usually affects thin women [40]. It occurs most frequently during pregnancy due to the hormonal changes with ovarian vein dilatation, valvular incompetence, and decreased muscular ureteral tone [40]. OVS should be differentiated from pelvic congestion syndrome, which consists of the dilatation of the entire anastomotic pathway of the pelvic venous system (ovarian, uterine, iliac), frequently with caudal extension to the lower extremity veins [41].

Obstruction from ureteral compression can be asymptomatic or clinically manifest with flank pain, hematuria, or pyelonephritis [1].

Contrast-enhanced CT should be acquired in both arterial, venous, and excretory phases for the correct evaluation of the ureter and adjacent vessels. The excretory phase images allow the visualization of ureteral compression by crossing vessels, with associated hydronephrosis and proximal dilatation of the ureter above the level of obstruction, while the caliber of the distal ureter is normal. These findings are better depicted on coronal images. However, the presence of ureter obstruction and hydronephrosis is not sufficient to make the diagnosis of ureteral vascular compression

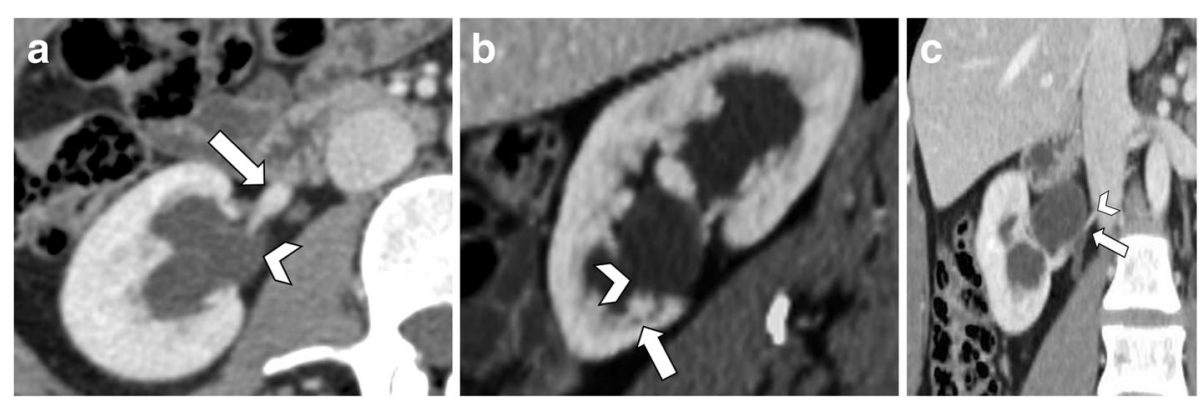

Fig. 13 A 31-year-old woman with ureteropelvic junction obstruction. a Axial CT image shows pelvic dilatation (arrowhead) caused by ureteropelvic junction compression by lower pole segmental renal artery (arrow). b The image reconstructed through an oblique sagittal plane, known as "hilar clock-face view", obtained orthogonal to the renal hilum, shows the relationship between the lower pole segmental renal artery (arrow) and the ureteropelvic junction (arrowhead). c Coronal CT image on the portal venous phase shows the ureteropelvic junction compressed (arrow) by lower pole segmental renal artery originating from the abdominal aorta (arrowhead) 


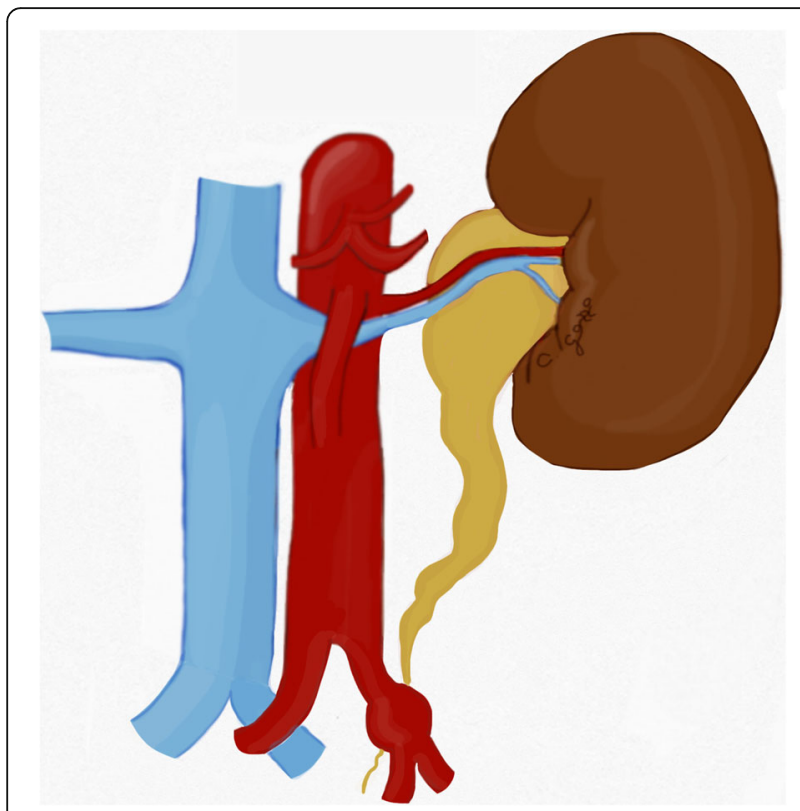

Fig. 14 Drawing illustrating the coronal view of ureteral obstruction by common iliac artery aneurysm

syndrome. Contrast-enhanced CT images on arterial or venous phase need to depict the presence of a common iliac artery aneurysm at the level of the iliac vessels (Fig. 16) or a dilated ovarian vein (Fig. 17) causing ureteral obstruction and exclude other causes of obstruction such as urinary calculi or tumoral strictures.

In symptomatic patients, surgical intervention to relieve the obstruction includes laparoscopic ureteroureterostomy, in case of retrocaval ureters, and ovarian vein ligation [1]. Transcatheter ovarian vein

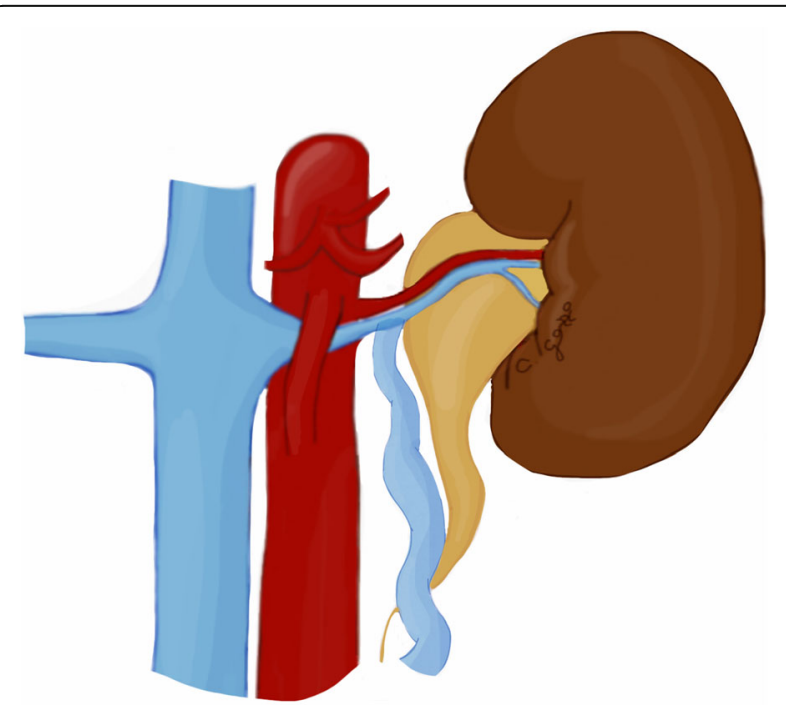

Fig. 15 Drawing illustrating the coronal view of left ovarian vein syndrome

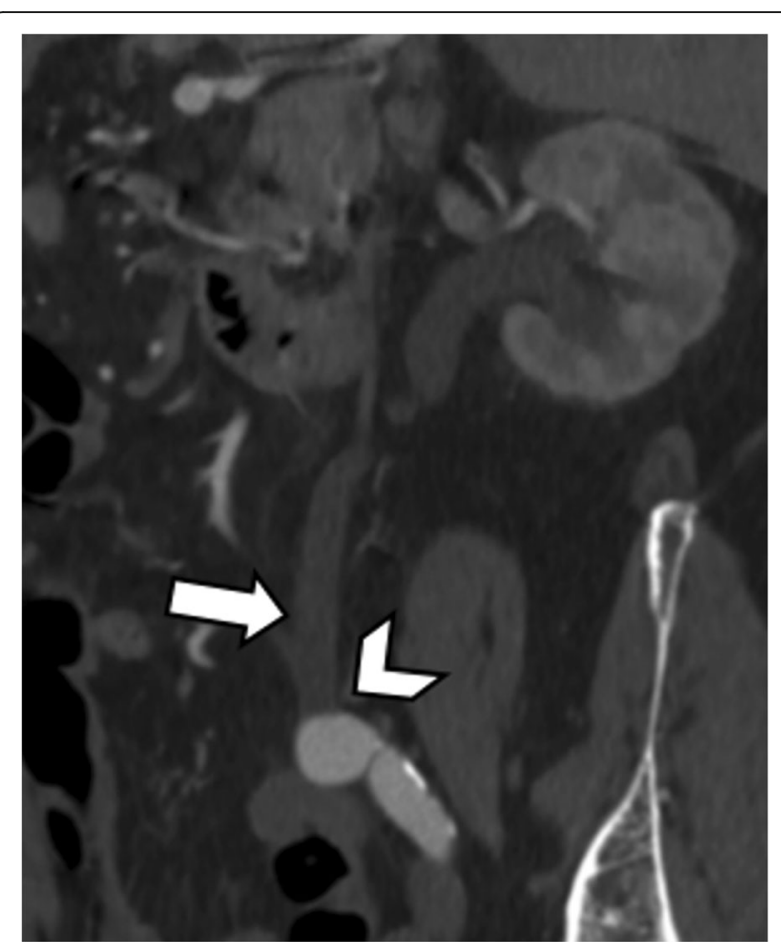

Fig. 16 An 83-year-old man with ureteral vascular compression. Sagittal CT image on arterial phase shows right hydroureteronephrosis (arrow) secondary to compression by a saccular aneurysm (arrowhead) of the adjacent right common iliac artery

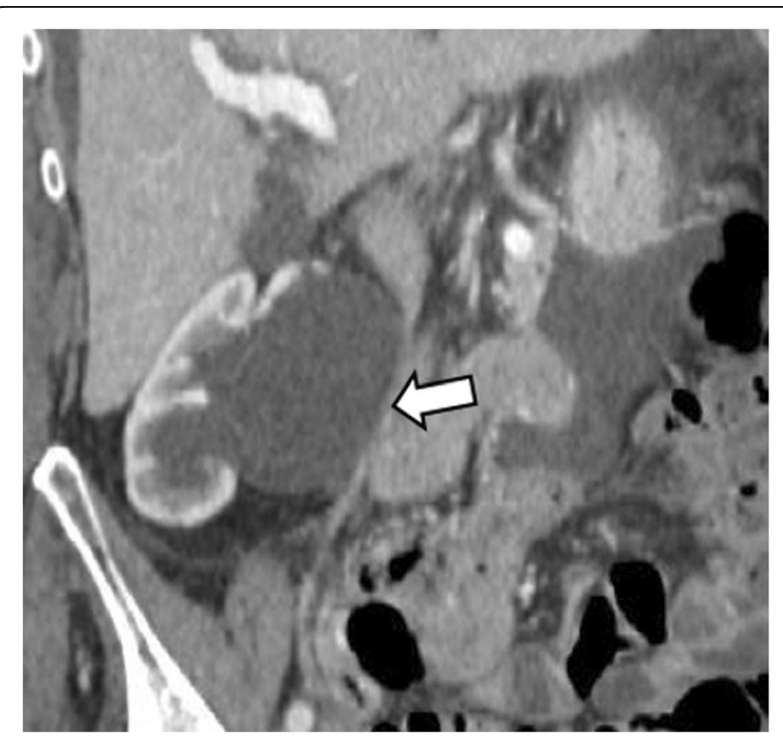

Fig. 17 An 84-year-old woman with upper ureteral vascular compression. Coronal enhanced CT image shows the proximal ureter (arrow) compressed by a dilated ovarian vein 


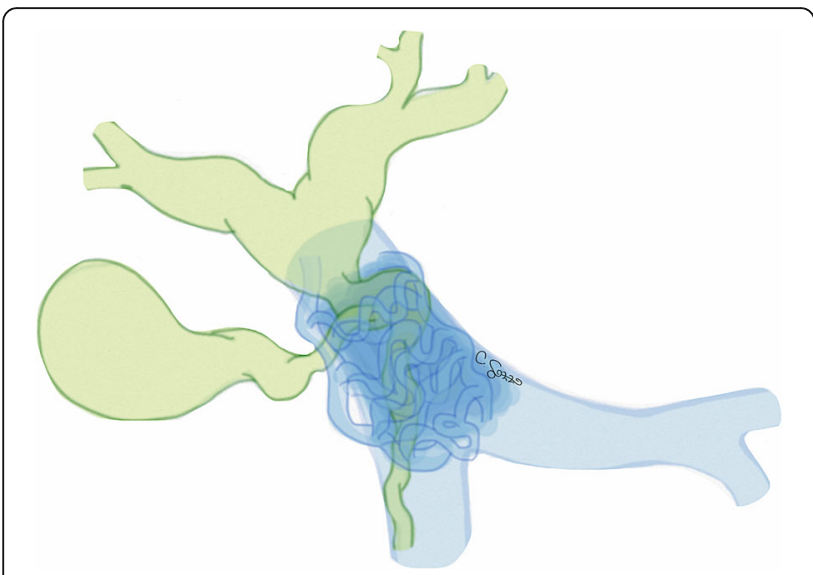

Fig. 18 Drawing illustrating the coronal view of portal biliopathy

embolization can be a minimally invasive option in OVS resistant to medical therapy [41].

\section{Portal biliopathy}

Portal biliopathy is defined as an abnormal dilatation of the extra- and/or intrahepatic biliary ducts, as well as the cystic duct and gallbladder, due to the presence of chronically thrombosed portal system evolved into "portal cavernoma" [42-44]. Portal cavernoma (Fig. 18) is the cavernous transformation of the venous drainage of the common bile duct formed by the paracholedochal veins (plexus of Petren) and the epicholedochal veins (plexus of Saint), located along the ductal wall [45]. There are two mechanisms responsible for portal biliopathy: an outer mechanical compression of the biliary system by portal cavernous transformation and/or peribiliary fibrosis secondary to inflammatory or ischemic changes caused by thrombosis of small veins in the bile duct walls [44, 46]. Indeed, portal biliopathy has been classified into three types: varicoid, fibrotic, and mixed. The varicoid type is the compression and distortion of the bile duct caused by large collateral veins (paracholedochal veins). The fibrotic type shows the tricking and narrowing of the bile duct, resulting from compression of smaller intramural collateral veins (epicholedochal veins) [46]. Chandra et al. [45] proposed an additional classification system according to the location of biliary obstruction with various combinations of extrahepatic, intrahepatic, unilateral, or bilateral intrahepatic ducts involvement.

About $70-100 \%$ of patients with radiological evidence of portal biliopathy are initially asymptomatic [44]. Portal biliopathy becomes clinically manifest only in a minority of patients with chronic cholestasis and jaundice [42, 43, 46]. Overall, 4-10\% patients may develop complications like choledocholithiasis, cholangitis, and secondary biliary cirrhosis [47].

Ultrasound may demonstrate the intra- and extrahepatic bile duct dilatation and the imaging findings of portal cavernous transformation [46], including multiple anechoic tubular structures with vascular flow at color Doppler, corresponding to paracholedochal collateral veins in hepatoduodenal ligament and porta hepatis, and increased flow in hepatic artery as a compensatory mechanism [46]. Contrast-enhanced CT (Fig. 19) is mainly performed to exclude other causes of biliary dilatation since it can mimic malignant strictures of the distal bile duct from pancreatic adenocarcinoma or biliary neoplasms [43]. The most common imaging finding of portal biliopathy, besides the portal cavernoma, is the acute angulation of the common bile duct forming a "kinking", which can be considered the consequence of the extrinsic compression by the dilated paracholedochal veins [48]. When MRI with cholangiography is performed, it may demonstrate a "scalloping" or "wavy" delineation of the extrahepatic biliary ducts secondary to indentations by the dilated peribiliary veins [48].

Portal biliopathy does not require specific treatment in asymptomatic patients. Symptomatic subjects may be approached with interventional radiology or surgical management [43]. Interventional radiology plays a role in biliary drainage, through the placement of nasobiliary or biliary stent, or in portal vein recanalization and transjugular intrahepatic portosystemic shunt (TIPS) placement in selected patients with chronic portal vein thrombosis $[49,50]$. In the case of

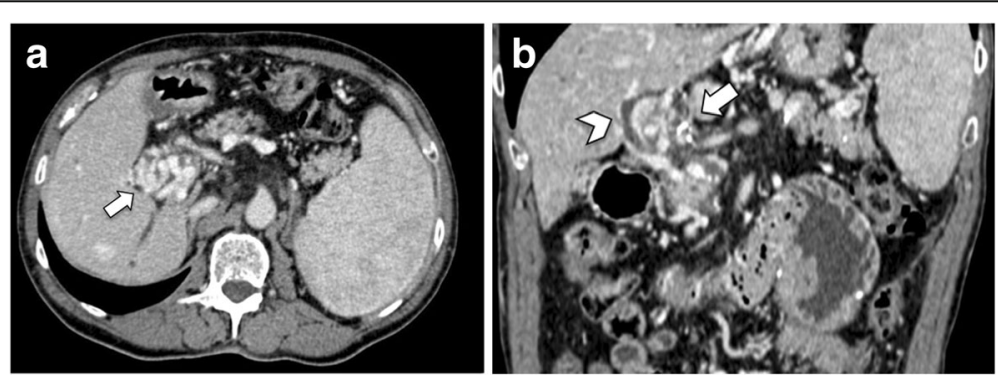

Fig. 19 A 63-year-old woman with portal biliopathy. a Axial enhanced CT image shows the cavernous transformation of the portal vein (arrow). $\mathbf{b}$ Coronal CT image demonstrates biliary ducts dilatation (arrowhead) due to compression by portal cavernoma (arrow) 
persistent biliary obstruction, a surgical decompression may be performed with hepaticojejunostomy or choledochoduodenostomy [43].

\section{Conclusions}

Abdominopelvic vascular compression syndromes may cause a wide spectrum of atypical abdominal symptoms or acute complications. Anatomical findings predisposing to these compression syndromes may be discovered even in asymptomatic subjects who undergo imaging for unrelated indications. In patients with suspected vascular compression syndrome, the combination of clinical symptoms, appropriate imaging modalities, and knowledge of typical imaging features is important for their accurate diagnosis and to guide the appropriate management. Particularly, contrastenhanced CT may be performed with different protocols according to the specific syndrome in order to maximize the visibility of involved vessels and allow the identification of typical imaging features and possible complications.

\begin{abstract}
Abbreviations
AMA: Aortomesenteric angle; CT: Computed tomography; LRV: Left renal vein; MALS: Median arcuate ligament syndrome; MRI: Magnetic resonance imaging; MTS: May-Thurner syndrome; NCS: Nutcracker syndrome; OVS: Ovarian vein syndrome; SMA: Superior mesenteric artery; TIPS: Transjugular intrahepatic portosystemic shunt; UPJO: Ureteropelvic junction obstruction
\end{abstract}

\section{Authors' contributions}

CG was the major contributor in writing and revising the manuscript, drawn illustrations. DG and RC substantially contributed to the work design, image collection, drafting, and editing of the manuscript. GC and AJ contributed to the literature research, images preparation, and made substantial manuscript editing. DP made substantial contribution to design and images collection. MM and GS revised the manuscript critically for important intellectual content. All authors read and approved the final manuscript.

\section{Funding}

The authors state that this work has not received any funding.

\section{Ethics approval and consent to participate}

Not applicable.

\section{Consent for publication}

Not applicable.

\section{Competing interests}

The authors declare that they have no competing interests.

\section{Author details \\ 'Sezione di Scienze Radiologiche, Biomedicina, Neuroscienze e Diagnostica avanzata (BIND), University of Palermo, Via del Vespro 129, 90127 Palermo, Italy. ${ }^{2}$ Department of Medical Surgical Sciences and Advanced Technologies "G.F. Ingrassia"-Radiology I Unit, University Hospital "Policlinico Vittorio Emanuele", Catania, Italy. ${ }^{3}$ Radiology Research Laboratory, Riga Stradins University, Riga, Latvia. ${ }^{4}$ Department of Pathology, Riga Stradins University, Riga, Latvia.}

Received: 8 November 2019 Accepted: 21 February 2020

Published online: 17 March 2020

\section{References}

1. Lamba R, Tanner D, Sekhon S, McGahan J, Corwin M, Lall C (2014) Multidetector $C T$ of vascular compression syndromes in the abdomen and pelvis. Radiographics 34:93-115
2. Eliahou R, Sosna J, Bloom Al (2012) Between a rock and a hard place: clinical and imaging features of vascular compression syndromes. Radiographics 32:E33-E49

3. Kim EN, Lamb K, Relles D, Moudgill N, DiMuzio PJ, Eisenberg JA (2016) Median Arcuate Ligament Syndrome-Review of This Rare Disease. JAMA Surg 151:471-477

4. Nasr LA, Faraj WG, Al-Kutoubi A et al (2017) Median Arcuate Ligament Syndrome: A Single-Center Experience with 23 Patients. Cardiovasc Intervent Radiol 40:664-670

5. Fong JK, Poh AC, Tan AG, Taneja R (2014) Imaging findings and clinical features of abdominal vascular compression syndromes. AJR Am J Roentgenol 203:29-36

6. Heo S, Kim HJ, Kim B, Lee JH, Kim J, Kim JK (2018) Clinical impact of collateral circulation in patients with median arcuate ligament syndrome. Diagn Interv Radiol 24:181-186

7. Jimenez JC, Harlander-Locke M, Dutson EP (2012) Open and laparoscopic treatment of median arcuate ligament syndrome. J Vasc Surg 56:869-873

8. Scholbach T (2006) Celiac artery compression syndrome in children, adolescents, and young adults: clinical and color duplex sonographic features in a series of 59 cases. J Ultrasound Med 25:299-305

9. Mak GZ, Speaker C, Anderson K et al (2013) Median arcuate ligament syndrome in the pediatric population. J Pediatr Surg 48:2261-2270

10. Sugae T, Fujii T, Kodera $Y$ et al (2012) Classification of the celiac axis stenosis owing to median arcuate ligament compression, based on severity of the stenosis with subsequent proposals for management during pancreatoduodenectomy. Surgery 151:543-549

11. Chincarini M, Zamboni GA, Pozzi Mucelli R (2018) Major pancreatic resections: normal postoperative findings and complications. Insights Imaging 9:173-187

12. Caruana G, Cannella R, Giambelluca D et al (2019) Pancreaticoduodenal arteries aneurysms: evaluation of frequency and association with celiac artery stenosis due to compression by median arcuate ligament. EuroMediterranean Biomedical Journal 14:101-106

13. Yun SJ, Lee JM, Nam DH, Ryu JK, Lee SH (2016) Discriminating renal nutcracker syndrome from asymptomatic nutcracker phenomenon using multidetector computed tomography. Abdom Radiol (NY) 41:1580-1588

14. Butros SR, Liu R, Oliveira GR, Ganguli S, Kalva S (2013) Venous compression syndromes: clinical features, imaging findings and management. $\mathrm{Br} J$ Radiol 86:20130284

15. Ananthan K, Onida S, Davies AH (2017) Nutcracker syndrome: an update on current diagnostic criteria and management guidelines. Eur J Vasc Endovasc Surg 53:886-894

16. Bookwalter CA, VanBuren WM, Neisen MJ, Bjarnason H (2019) Imaging appearance and nonsurgical management of pelvic venous congestion syndrome. Radiographics 39:596-608

17. Singla RK, Sharma T, Gupta R (2010) Retro-aortic left renal vein with left suprarenal vein draining into inferior vena cava. IJAV 3:134-137

18. Kim KW, Cho JY, Kim SH et al (2011) Diagnostic value of computed tomographic findings of nutcracker syndrome: Correlation with renal venography and renocaval pressure gradients. Eur J Radiol 80:648-654

19. Merrett ND, Wilson RB, Cosman P, Biankin AV (2009) Superior mesenteric artery syndrome: diagnosis and treatment strategies. J Gastrointest Surg 13:287-292

20. Warncke ES, Gursahaney DL, Mascolo M, Dee E (2019) Superior mesenteric artery syndrome: a radiographic review. Abdom Radiol (NY) 44:3188-3194

21. He Y, Wu Z, Chen S et al (2014) Nutcracker syndrome-How well do we know it? Urology 83:12-17

22. Kim WS, Cheon J-E, Kim IO et al (2006) Hemodynamic Investigation of the left renal vein in pediatric varicocele: Doppler US, venography, and pressure measurements. Radiology 241:228-234

23. Shin Jl, Baek SY, Lee JS, Kim MJ (2007) Follow-up and treatment of nutcracker syndrome. Ann Vasc Surg 21:402

24. Erben Y, Gloviczki P, Kalra M et al (2015) Treatment of nutcracker syndrome with open and endovascular interventions. J Vasc Surg Venous Lymphat Disord 3:389-396

25. Wang X, Zhang Y, Li C, Zhang H (2012) Results of endovascular treatment for patients with nutcracker syndrome. J Vasc Surg 56:142-148

26. Morita S1, Kimura T, Masukawa A, Saito N, Suzuki K, Mitsuhashi N (2007) Flow direction of ascending lumbar veins on magnetic resonance angiography and venography: would "descending lumbar veins" be a more precise name physiologically? Abdom Imaging 32:749-753. 
27. Mousa AY, AbuRahma AF (2013) May-Thurner syndrome: update and review. Ann Vasc Surg 27:984-995

28. ELsaid AS, AlQattan AS, Elashaal E, AISadery H, AlGhanmi I, Aldhafery BF (2019) The ugly face of deep vein thrombosis: Phlegmasia Cerulea DolensCase report. Int J Surg Case Rep 59:107-110

29. Heller T, Teichert C, Hafer J, Weber MA, Kröger JC, Meinel FG (2019) Prevalence of May-Thurner syndrome in patients with deep vein thrombosis at a Large Medical Referral Center. Rofo. https://doi.org/10.1055/a-0959-6230

30. Moudgill N, Hager E, Gonsalves C, Larson R, Lombardi J, DiMuzio P (2009) May-Thurner syndrome: case report and review of the literature involving modern endovascular therapy. Vascular 17:330-335

31. Ganss A, Rampado S, Savarino E, Bardini R (2019) Superior mesenteric artery syndrome: a prospective study in a single institution. J Gastrointest Surg 23: 997-1005

32. Sinagra E, Raimondo D, Albano D et al (2018) Superior mesenteric artery syndrome: clinical, endoscopic, and radiological findings. Gastroenterol Res Pract 2018:1937416

33. Mathenge N, Osiro S, Rodriguez II, Salib C, Tubbs RS, Loukas M (2014) Superior mesenteric artery syndrome and its associated gastrointestinal implications. Clin Anat 27:1244-1252

34. Horvat N, Brentano VB, Abe ES, Dumarco RB, Viana PCC, Machado MCC (2019) A rare case of idiopathic congenital megaduodenum in adult misinterpreted during childhood: case report and literature review. Radiol Case Rep 14:858-863

35. Cancian M, Pareek G, Caldamone A, Aguiar L, Wang H, Amin A (2017) Histopathology in ureteropelvic junction obstruction with and without crossing vessels. Urology 107:209-213

36. Lawler LP, Jarret TW, Corl FM, Fishman EK (2005) Adult ureteropelvic junction obstruction: insights with three-dimensional multi-detector row $\mathrm{CT}$. Radiographics 25:121-134

37. Calder AD, Hiorns MP, Abhyankar A, Mushtaq I, Olsen OE (2007) Contrastenhanced magnetic resonance angiography for the detection of crossing renal vessels in children with symptomatic ureteropelvic junction obstruction: comparison with operative findings. Pediatr Radiol 37:356-361

38. Magliocca JK, Faerber GJ, Gilbert R (2005) Solitary common iliac artery inflammatory aneurysm in a healthy woman: case report and review of the literature. Ann Vasc Surg 19:890-895

39. Cox A, Patel S, Kumaradevan J (2016) Renal colic caused by mycotic iliac artery aneurysm. BJR Case Rep 2:20150155

40. Manoharan V, Parmar K, Mavuduru RS, Rai T, Tyagi S (2019) A rare indication of robot-assisted uretero-ureterostomy: ovarian vein syndrome. J Robot Surg 13:703-705

41. Bhutta HY, Walsh SR, Tang TY, Walsh CA, Clark JM (2009) Ovarian vein syndrome: a review. Int J Surg 7:516-520

42. Shin SM, Kim S, Lee JW et al (2007) Biliary abnormalities associated with portal biliopathy: evaluation on MR cholangiography. AJR Am J Roentgenol 188:W341-W347

43. Dhiman RK, Saraswat VA, Valla DC et al (2014) Portal cavernoma cholangiopathy: consensus statement of a working party of the Indian national association for study of the liver. J Clin Exp Hepatol 4:S2-S14

44. Moomjian LN, Winks SG (2017) Portal cavernoma cholangiopathy: diagnosis, imaging, and intervention. Abdom Radiol (NY) 42:57-68

45. Chandra R, Kapoor D, Tharakan A, Chaudhary A, Sarin SK (2001) Portal biliopathy. J Gastroenterol Hepatol 16:1086-1092

46. Srisajjakul S, Prapaisilp P, Bangchokdee S (2017) Imaging features of vascular compression in abdomen: fantasy, phenomenon, or true syndrome. Indian J Radiol Imaging 27:216-224

47. Khanna R, Sarin SK (2018) Idiopathic portal hypertension and extrahepatic portal venous obstruction. Hepatol Int 12:148-167

48. Jabeen S, Robbani I, Choh NA et al (2016) Spectrum of biliary abnormalities in portal cavernoma cholangiopathy (PCC) secondary to idiopathic extrahepatic portal vein obstruction (EHPVO) - a prospective magnetic resonance cholangiopancreaticography (MRCP) based study. Br J Radiol 89: 20160636

49. Thornburg B, Desai K1, Hickey R, et al (2016) Portal vein recanalization and transjugular intrahepatic portosystemic shunt creation for chronic portal vein thrombosis: technical considerations. Tech Vasc Interv Radiol 19:52-60.

50. Thornburg B, Desai K1, Hickey R, et al (2017) Pretransplantation portal vein recanalization and transjugular intrahepatic portosystemic shunt creation for chronic portal vein thrombosis: Final Analysis of a 61-Patient Cohort. J Vasc Interv Radiol 28:1714-1721.e2.

\section{Publisher's Note}

Springer Nature remains neutral with regard to jurisdictional claims in published maps and institutional affiliations.

\section{Submit your manuscript to a SpringerOpen ${ }^{\circ}$ journal and benefit from:}

- Convenient online submission

- Rigorous peer review

- Open access: articles freely available online

High visibility within the field

- Retaining the copyright to your article

Submit your next manuscript at $\boldsymbol{\nabla}$ springeropen.com 